\title{
Magnetization dynamics down to zero field in dilute (Cd,Mn)Te quantum wells
}

\author{
M. Goryca ${ }^{1}$, D. Ferrand ${ }^{2}$, P. Kossacki ${ }^{1,2}$, M. Nawrocki ${ }^{1}$, W. Pacuski ${ }^{1,2}$, W. Maślana ${ }^{1,2}$, \\ J.A. Gaj ${ }^{1}$, S. Tatarenko ${ }^{2}$, J. Cibert ${ }^{2}$, T. Wojtowicz ${ }^{3}$, and G. Karczewski ${ }^{3}$ \\ 1 Institute of Experimental Physics, University of Warsaw, Hoża 69, PL-00-681 Warszawa, Poland. \\ 2 Institut Néel, CNRS/UJF, BP166, F-38042 Grenoble Cedex 9, France. \\ 3 Institute of Physics, Polish Academy of Sciences, Warsaw, Poland
}

(Dated: October 17, 2008)

\begin{abstract}
The evolution of the magnetization in (Cd,Mn)Te quantum wells after a short pulse of magnetic field was determined from the giant Zeeman shift of spectroscopic lines. The dynamics in absence of magnetic field was found to be up to three orders of magnitude faster than that at $1 \mathrm{~T}$. Hyperfine interaction and strain are mainly responsible for the fast decay. The influence of a hole gas is clearly visible: at zero field anisotropic holes stabilize the system of Mn ions, while in a magnetic field of $1 \mathrm{~T}$ they are known to speed up the decay by opening an additional relaxation channel.
\end{abstract}

The quest for carrier-induced ferromagnetism, and for systems appropriate to spin manipulation in the context of quantum information processing, stimulates intense studies of Diluted Magnetic Semiconductors (DMS) [1, 2, 3. In the DMS family, (Cd,Mn)Te appears as particularly suitable for studies of low dimensional structures, from quantum wells (QWs) exhibiting carrierinduced ferromagnetic interactions [4], to quantum dots (QDs) containing a single magnetic impurity [5]. This flexibility is due to the isoelectronic character of $\mathrm{Mn}$ in $\mathrm{CdTe}$, offering the possibility of modulation doping and electrical biasing of nanostructures [6, 77, and to the broad range of optical methods available, which yield direct information on the magnetic configuration of the Mn system through the so called giant Zeeman effect. While the static properties of these nanostructures are essentially well understood, new questions arise about the magnetization dynamics. In particular, understanding spin dynamics in absence of any applied magnetic field is urgently needed.

Spin dynamics of $\mathrm{Mn}$ in $(\mathrm{Cd}, \mathrm{Mn}) \mathrm{Te}$ has been studied, for years, almost exclusively in the presence of a magnetic field. Typical measurements involve a short heat pulse used to drive the sample out of thermal equilibrium, the evolution of the magnetization being measured with a pick-up coil [8] or extracted from the giant Zeeman effect in photoluminescence (PL) [9]. Faraday rotation following the creation of electron-hole pairs by a laser pulse reveals the transverse relaxation time $T_{2}$ 10, 11. $T_{2}$ was also deduced from the width of the Electron Paramagnetic Resonance (EPR) line, in the range of Mn content where it exhibits exchange narrowing.

The relaxation of the transverse component of magnetization is an adiabatic process, which was ascribed to anisotropic spin-spin interactions [12]. It is fast, and accelerates with the Mn content. The relaxation of the longitudinal component involves a transfer of the Zeeman energy to the lattice (spin-lattice relaxation). For isolated $\mathrm{Mn}^{2+}$ ions, this is a slow process, due to the absence of spin-orbit coupling within the orbital singlet which forms the ground state. In DMSs, the spin-lattice

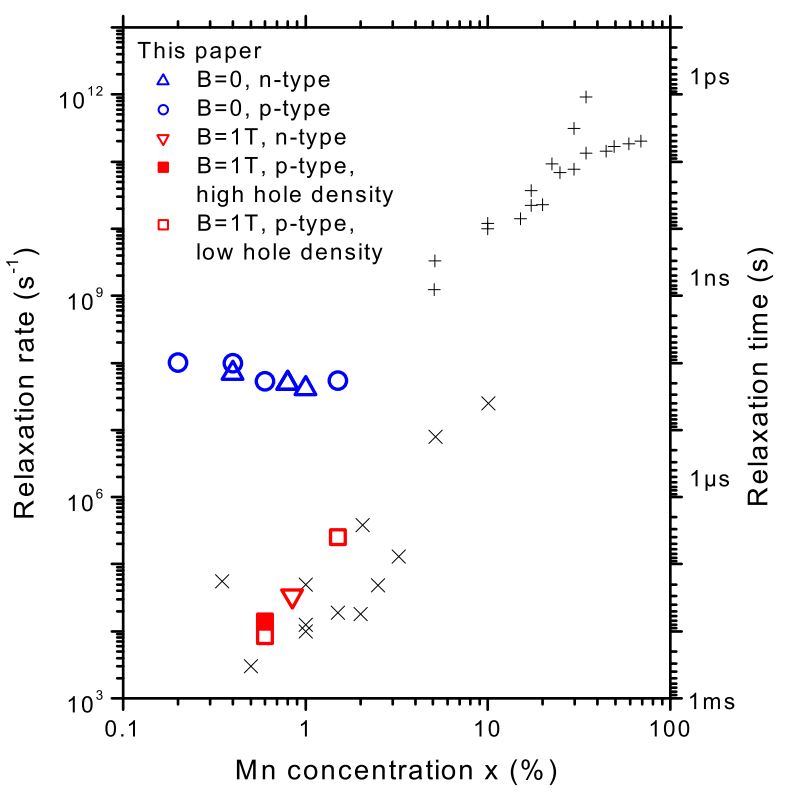

FIG. 1: spin-spin relaxation rate at $5 \mathrm{~K}(+)$, and spin - lattice relaxation at $1.5 \mathrm{~K}$ to $4.7 \mathrm{~K}(\times)$, and present data: fast and slow magnetization decay at $0 \mathrm{~T}$ and $1 \mathrm{~T}$, respectively (open symbols, carrier densities in the $10^{10} \mathrm{~cm}^{-2}$ range, closed symbol $\left.3 \times 10^{11} \mathrm{~cm}^{-2}\right)$. Adapted from Ref. 114.

relaxation rate increases with the $\mathrm{Mn}$ content, as a result of a fast transfer via the Mn spins towards "killer centers", in particular Mn clusters [13] which statistically exist within the random Mn distribution. For compositions where both have been measured, the spin-spin time is faster than the spin-lattice relaxation time, by 2 to 3 orders of magnitude (see Fig. 1, adapted from Ref. [14]). The general picture that emerges, is that fast processes are expected in a DMS with a high Mn content, while strongly diluted or isolated Mn spins should exhibit long relaxation times. More recently [9], the role of free carriers has been stressed: they form an efficient channel to transfer the Mn Zeeman energy to the lattice.

Experimental data at zero field are scarce. In Ref. [15], the magnetization of a bulk $(\mathrm{Cd}, \mathrm{Mn}) \mathrm{Te}$ sample, in the 
presence of a static field, was changed by the collinear, AC field of a coil, and monitored by Faraday rotation. The spin-lattice relaxation time was clearly identified and exhibits a dramatic decrease at low field. In principle, this method can be used down to zero field but the frequency response of the set-up was limited to a few microseconds, so that the static field had to be kept above a few tenths of tesla. By contrast, $T_{2}$ slowly increases when decreasing the applied field in $\mathrm{Zn}_{0.9} \mathrm{Mn}_{0.1}$ Se [10] or $(\mathrm{Cd}, \mathrm{Mn}) \mathrm{Te}$ [1].

In this paper we describe the magnetization dynamics after a short magnetic pulse created by a small coil, down to zero field and with a temporal resolution of a few ns, in (Cd,Mn)Te QWs with low Mn content, and various densities of electrons or holes.

Samples contain a single 8 nm-wide, $p$-type or $n$ type, (Cd,Mn)Te QW, with $0.2 \%$ to $1.5 \% \mathrm{Mn}$, and (Cd,Mg,Zn)Te barriers. The whole structure was grown coherently, by molecular beam epitaxy, on a $\mathrm{Cd}_{0.96} \mathrm{Zn}_{0.04} \mathrm{Te}$ or $\mathrm{Cd}_{0.88} \mathrm{Zn}_{0.12}$ Te substrate or a $\mathrm{CdTe}$ buffer layer grown on GaAs. Holes were introduced into the QW either by modulation doping with Nitrogen or by tunneling from surface electron traps [16]. Electrons were provided by modulation doping with Iodine 17 . The carrier density ranged from $1 \times 10^{10}$ to $3 \times 10^{11} \mathrm{~cm}^{-2}$.

Pulses of magnetic field were produced with a small coil (28 turns, internal diameter $400 \mu \mathrm{m}$ ) mounted directly on the sample surface, fed by a coaxial transmission line terminated with a matched resistor. A 2 A current produces $\approx 40 \mathrm{mT}$, as calibrated on PL spectra of samples with a high Mn content [18], with rise and fall times $\approx 7 \mathrm{~ns}$ (see Fig. 2). Illumination (a cw linearly polarized diode laser) and collection of PL signal (time resolved photon counting system) were done through the aperture of the coil, along its axis, and in some cases a static magnetic field was added along the same axis (Faraday configuration). Fig. 2a displays PL spectrum of a $p$-type sample, obtained under typical conditions used in the present study. It features a single line related to the charged exciton $X^{+}$(at this carrier density, the neutral exciton is not visible). Application of a magnetic field results in a giant Zeeman shift opposite in the two circular polarizations, proportional to the magnetization change. If the Zeeman shift induced by the field pulse is much smaller than the linewidth, by measuring the intensity difference between the two polarizations, we obtain a signal proportional to the Mn magnetization (Fig. 2b). A higher dynamics (3 orders of magnitude) but a slightly lower time resolution ( $\approx 20 \mathrm{~ns}$ ) was obtained using transmission or reflectivity. Then, both the charged and neutral exciton lines are visible, so that the field pulse induces not only a shift, but also intensity transfers [19]. The setup for transmission was the same as that for PL, but with a halogen lamp. In reflectivity, we used light pulses synchronized with the field pulse, either from a cw Ti:sapphire laser modulated to pulses shorter than $20 \mathrm{~ns}$ using an acousto-optic mod-

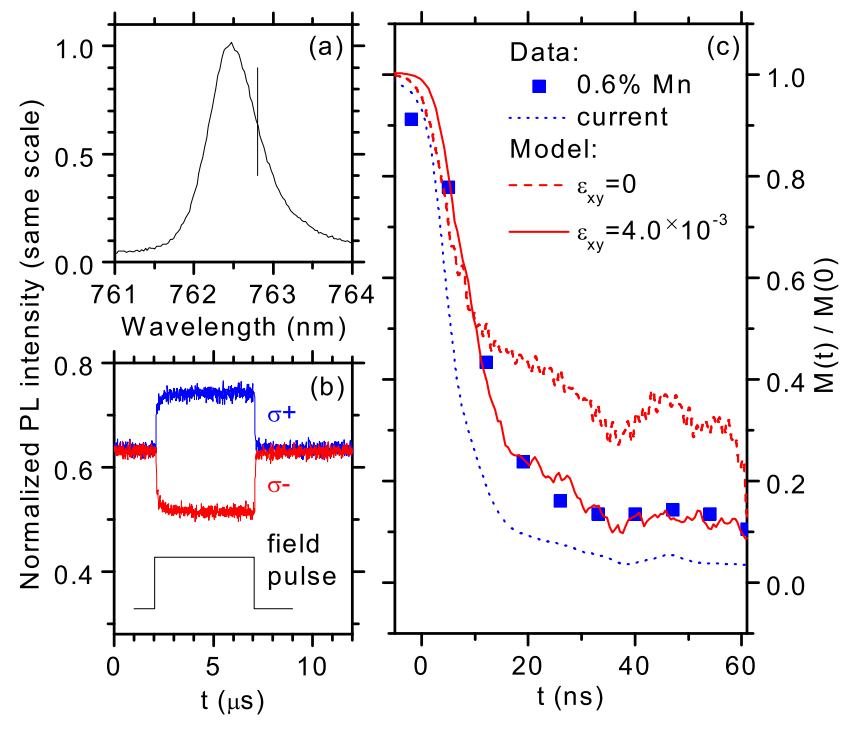

FIG. 2: PL at $B=0$ for a QW with $0.6 \% \mathrm{Mn}$ and $p \approx$ $10^{10} \mathrm{~cm}^{-2}$. (a) Spectrum; (b) intensity vs. time during a field pulse, at the wavelength marked with a vertical line in (a). (c) Magnetization dynamics (symbols), coil current (dotted line) and result of calculations (solid and dashed lines) as described in the text.

ulator, or from a pulsed Ti:sapphire laser. An optical bridge was used to monitor the difference between the two circular polarizations of the reflected light.

Fig. 2 2 c shows an example of the PL signal observed without applied static field. A bi-exponential decay convoluted with the current profile describes well the magnetization decay. The faster characteristic time is about $20 \mathrm{~ns}$, and it is longer than the experimental resolution $(\approx 7 \mathrm{~ns})$. It is smaller than the values obtained in magnetic field by about three orders of magnitude. It shows almost no dependence on the Mn content (Fig. 1), as expected for isolated Mn ions.

Several interactions may be responsible for the fast zero-field decay observed: the hyperfine interaction with the nuclear spin of the Mn ion itself, the superhyperfine interaction with the nuclear spin of neighbor Cd atoms, dipolar interaction with nuclear spins or spins of other Mn ions, the single ion anisotropy due to the cubic crystal field and to strain in the epitaxial structure, fluctuations in the interaction with carriers, and so on...

It is well known, from EPR spectroscopy of Mn diluted in CdTe- and ZnTe-MnTe superlattices 20, 21], that the Mn electronic spin $\vec{S}$ interacts strongly with its nuclear spin $\vec{I}$ (both 5/2), with a sizable crystal field, and with strain. The Hamiltonian assessed by EPR is

$$
\mathcal{H}=g \mu_{B} \vec{B} \cdot \vec{S}+A \vec{I} \cdot \vec{S}+D\left[S_{z}^{2}-\frac{S(S+1)}{3}\right]
$$




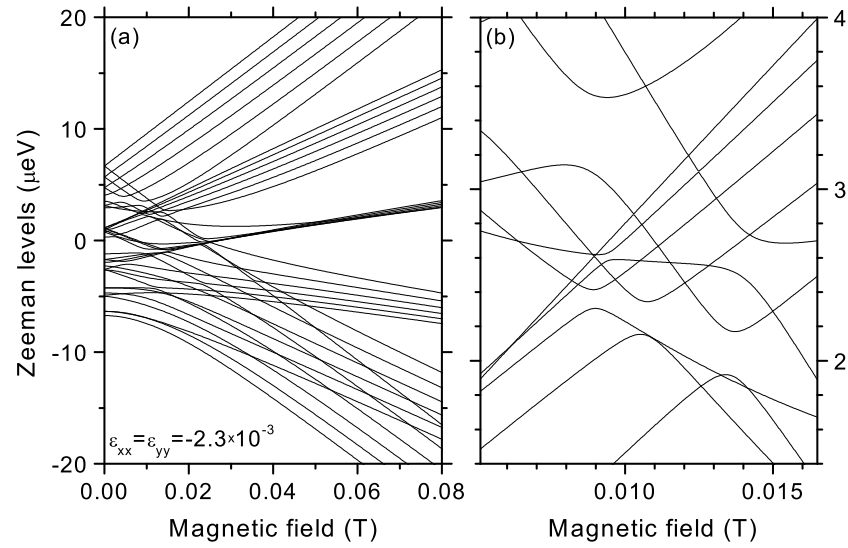

FIG. 3: Energy levels taking into account the hyperfine coupling, cubic crystal field and mismatch strain.

$$
+\frac{a}{6}\left[S_{x}^{4}+S_{y}^{4}+S_{z}^{4}-\frac{S(S+1)\left(3 S^{2}+3 S-1\right)}{5}\right]
$$

with $A=680 \mathrm{neV}$ for the hyperfine coupling, $a=320 \mathrm{neV}$ for the cubic crystal field. The dominant strain component in epitaxial layers grown pseudomorphically on substrates with $4 \%$ and $12 \% \mathrm{Zn}$ is biaxial, with $\varepsilon_{x x}=\varepsilon_{y y}=-\left(c_{11} / 2 c_{12}\right) \varepsilon_{z z}=-2.3 \times 10^{-3}$ and $-7 \times 10^{-3}$, respectively. Its effect is described by the $D S_{z}^{2}$ term, with values of $D$ deduced from Ref. [21]. A typical energy diagram is shown in Fig. 3 .

Strong anticrossings appear at low values of the static field (Fig. 3 $\mathrm{b}$ ): therefore sweeping down the magnetic field at the end of the pulse produces a change not only in the Zeeman energies but also in the state of the Mn. The evolution of the magnetization can be viewed as a series of Landau-Zener processes; it is easily obtained by solving numerically the Schrödinger equation of the density matrix, with an initial value corresponding to a thermalized system. As shown by the dashed line in Fig. 2cc, this calculation of the evolution of the coupled Mn electronic and nuclear spins, averaged over a thermal distribution but with no additional effect of the environment, well describes the first fast drop in the experimental data.

The hyperfine coupling has non-zero matrix elements only between states with $\Delta S_{z}= \pm 1$. So several levels show no anticrossing, and the spin states remain eigenstates when varying the magnetic field. This results in the presence of a persistent magnetization which is clearly visible in Fig. 2.

The fast decay due to the hyperfine field and the persistent magnetization are not much changed when varying slightly the value of the biaxial strain, which in our samples remains quite low. A larger value of the strain would induce a splitting of the $S=5 / 2$ sextuplet into three $\pm m$ doublets, see Fig. 2 in Ref. [21]. In particular, around zero field, the $\pm 5 / 2$ and $\pm 3 / 2$ exhibit no anticrossing, only the $\pm 1 / 2$ doublet does. Such a strong strain exists in CdTe QD grown on ZnTe [5], with the

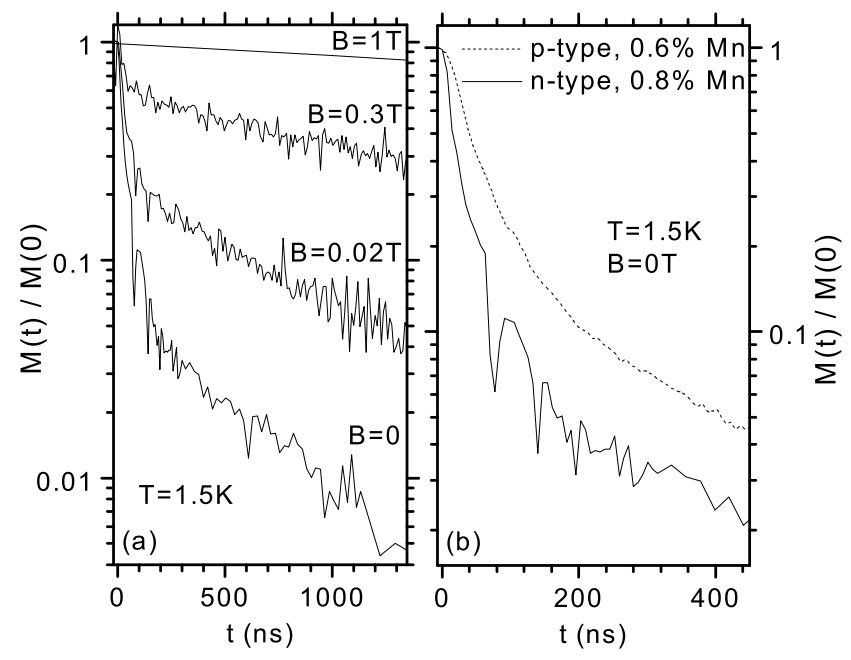

FIG. 4: (a) Magnetization decay in a sample with $0.8 \% \mathrm{Mn}$ and $n \approx 10^{10} \mathrm{~cm}^{-2}$ at indicated values of the static magnetic field. (b) Magnetization decay in $n$-type and $p$-type ( $p \approx$ $3 \times 10^{11} \mathrm{~cm}^{-2}$ ) samples at $0 \mathrm{~T}$.

\section{$\pm 1 / 2$ as the ground state.}

A more complete decay is calculated when introducing additional mechanisms which mix states with different values of $S_{z}$. Within the single Mn system, this is simulated when considering strain components which are anisotropic within the QW plane. For instance, the effect of a strain $\varepsilon_{x y}=4 \times 10^{-3}$, calculated using Ref. [22, 23], is shown in Fig. 20 as a solid line. It shows a good agreement with the experimental data. Such a strain in a QW could be caused by local fluctuations or dislocations, but its estimated value is surprisingly large: x-ray diffraction data gives a 4 times smaller estimate for similar structures [24]. This suggests that mechanisms related to the environment of the Mn impurity should be considered.

Note also that even for the best fit, the calculated evolution leaves a small but nonzero persistent magnetization. The experimental data in Fig. 2 $\mathrm{c}$ and ta $(B=0)$ shows indeed such a long-time component, which decays with characteristic times in sub- $\mu$ s range. Upon application of an external, static magnetic field, the characteristic time and the amplitude of this long-time component increase. A precise study of this effect is beyond the scope of the present work.

It is worth to note, however, that at field of $1 \mathrm{~T}$ the signal is mono-exponential, with a characteristic time in good agreement with previous data of spin-lattice relaxation in $(\mathrm{Cd}, \mathrm{Mn}) \mathrm{Te}$, see Fig. 11. In a $p$-type sample, the decay is faster for a larger hole density (which is easily controlled by additional illumination [25]), in agreement with Ref. 26]. Also the dependence of the decay at 1 $\mathrm{T}$ on the $\mathrm{Mn}$ content (Fig. 1) agrees with previous observations [14]. Actually, at $1 \mathrm{~T}$, the pulse field merely changes the Zeeman energy of the six sublevels of the 
$S=5 / 2$ multiplet, so that the populations have to readjust through spin-lattice relaxation [13].

As examplified in Fig. \$b, at the zero-field dynamics is systematically slower in the presence of holes than in the presence of electrons. We ascribe this effect to the anisotropy introduced by holes. Due to confinement, these are heavy holes with projections $\pm 3 / 2$ of their moment along the growth direction. Due to the strong exchange coupling between holes and Mn spins, the $\pm 5 / 2$ doublet of the Mn spin (with the proper orientation of the holes) forms the ground state, while the $\pm 3 / 2$ and $\pm 1 / 2$ ones are at higher energy. A polarized hole gas of density in the $10^{11} \mathrm{~cm}^{-2}$ range induces an exchange field of the order of $0.1 \mathrm{~T}$, i.e., a splitting of $10 \mu \mathrm{eV}$ between the $\pm 5 / 2$ and $\pm 3 / 2$ doublets. This results in an effective anisotropy similar to that occurring in molecular magnets, where it gives rise to a slow dynamics of the magnetization [27. Thus the effect of holes on the dynamics of the Mn spin at zero field (slowing down due to an effective anisotropy) is opposite to that at high field (additional relaxation channel). It was also pointed out that in a ferromagnetic (Cd,Mn)Te QW, this coupling results in a softening of the Mn resonance when approaching the critical temperature [28, 29], and a slow decay in the ferromagnetic phase [18].

To sum up, the use of a fast magnetic pulse reveals strong peculiarities of the Mn spin dynamics in very dilute DMSs. At zero magnetic field, a fast dynamics is observed. This dynamics is driven by hyperfine coupling with the nuclear spin of the Mn ion; it is highly sensitive to the presence of an anisotropy, particularly that introduced by holes and by strain. In the present study of an ensemble of Mn spins, with a thermal distribution of nuclear spins, this results in a fast decay of the magnetization when the field is swept down to zero. These mechanisms are expected to strongly influence the behavior of a single Mn spin, with different consequences for the dephasing time observed in time-averaged measurements and the coherence time in correlation experiments.

We thank Denis Scalbert, Lucien Besombes, Henryk Szymczak, and Bernard Barbara for fruitful discussions. This work was partially supported by the Polish Ministry of Science and Higher Education as research grants in years 2006-2009, Polonium and Marie-Curie programs and by the 6th Research Framework Programme of EU (contract MTKD-CT-2005-029671).

* mgoryca@fuw.edu.p

[1] H. Ohno, Science 281, 951 (1998).

[2] J. Fernández-Rossier and Ramón Aguado, Phys. Rev. Lett. 98, 106805 (2007).

[3] R. C. Myers, M. H. Mikkelsen, J. M. Tang, A. C. Gossard, M. E. Flatté, and D. D. Awschalom, Nature mat. 7, 1 (2008).
[4] A. Haury, A. Wasiela, A. Arnoult, J. Cibert, T. Dietl, Y. Merle d'Aubigné, and S. Tatarenko, Phys. Rev. Lett. 79, 511 (1997).

[5] L. Besombes, Y. Léger, L. Maingault, D. Ferrand, H. Mariette, and J. Cibert, Phys. Rev. Lett. 93, 207403 (2004).

[6] H. Boukari, P. Kossacki, M. Bertolini, D. Ferrand, J. Cibert, S. Tatarenko, A. Wasiela, J. A. Gaj, and T. Dietl, Phys. Rev. Lett. 88, 207204 (2002).

[7] Y. Léger, L. Besombes, J. Fernández-Rossier, L. Maingault, and H. Mariette, Phys. Rev. Lett. 97, 107401 (2006).

[8] T. Strutz, A. M. Witowski, and P. Wyder, Phys. Rev. Lett. 68, 3912 (1992).

[9] A. V. Scherbakov, A. V. Akimov, D. R. Yakovlev, W. Ossau, A. Waag, G. Landwehr, T. Wojtowicz, G. Karczewski, and J. Kossut, Phys. Rev. B 60, 5609 (1999).

[10] S. A. Crooker, J. J. Baumberg, F. Flack, N. Samarth, and D. D. Awschalom, Phys. Rev. Lett. 77, 2814 (1996).

[11] C. Camilleri, F. Teppe, D. Scalbert, Y. G. Semenov, M. Nawrocki, M. Dyakonov, J. Cibert, S. Tatarenko, and T. Wojtowicz, Phys. Rev. B 64, 085331 (2001); C. Camilleri, PhD thesis, Montpellier 2000.

[12] B. E. Larson and H. Ehrenreich, Phys. Rev. B 39, 1747 (1989).

[13] D. Scalbert, Phys. Stat. Sol. b 193, 189 (1996).

[14] T. Dietl, P. Peyla, W. Grieshaber, and Y. Merle d'Aubigné, Phys. Rev. Lett. 74, 474 (1995), and references therein.

[15] D. Scalbert, J. Cernogora, and C. Benoît à la Guillaume, Solid State Commun. 66, 571(1988).

[16] W. Maślana, M. Bertolini, H. Boukari, P. Kossacki, D. Ferrand, J. A. Gaj, S. Tatarenko, and J. Cibert, Appl. Phys. Lett. 82, 1875 (2003).

[17] T. Wojtowicz, M. Kutrowski, G. Karczewski, and J. Kossut, Appl. Phys. Lett. 73, 1379 (1998).

[18] P. Kossacki, D. Ferrand, M. Goryca, M. Nawrocki, W. Pacuski, W. Maślana, S. Tatarenko, and J. Cibert, Physica E 32, 454 (2006).

[19] P. Kossacki, J. Cibert, D. Ferrand, Y. Merle d'Aubigné, A. Arnoult, A. Wasiela, S. Tatarenko, and J. A. Gaj, Phys Rev B 60, 16018 (1999).

[20] J. Lambe, and C. Kikuchi, Phys. Rev. 119, 1256 (1960).

[21] M. Quazzaz, G. Yang, S. H. Xin, L. Montes, H. Luo, and J. K. Furdyna, Solid State Communic. 96, 405 (1995).

[22] Elsa Rosenvasser Feher, Phys. Rev. 136, A145 (1964).

[23] M. T. Causa, M. Tovar, S. B. Oseroff, R. Calvo, and W. Giriat, Physics Letters 77A, 473 (1980).

[24] C. Bodin, R. André, J. Cibert, Le Si Dang, D. Bellet, G. Feuillet, and P. H. Jouneau, Phys. Rev. B 51, 13181 (1995)

[25] P. Kossacki, H. Boukari, M. Bertolini, D. Ferrand, J. Cibert, S. Tatarenko, J. A. Gaj, B. Deveaud, V. Ciulin, and M. Potemski, Phys. Rev. B 70,195337 (2004).

[26] A. V. Scherbakov, A. V. Akimov, D. R. Yakovlev, W. Ossau, L. W. Molenkamp, S. Tatarenko, and J. Cibert, Solid State Commun. 120, 17 (2001).

[27] L. Bogani and W. Wernsdorfer, Nature Mat. 7, 179 (2008).

[28] K. V. Kavokin, Phys. Rev. B 59, 9822 (1999).

[29] D. Scalbert, F. Teppe, M. Vladimirova, S. Tatarenko, J. Cibert, and M. Nawrocki, Phys. Rev. B 70, 245304 (2004). 